\title{
AN APPLICATION OF MULTICRITERIA OPTIMIZATION TO THE TWO-CARRIER TWO-SPEED PLANETARY GEAR TRAINS
}

UDC 621.833.65-027.231

\author{
Jelena Stefanović-Marinović ${ }^{1}$, Sanjin Troha ${ }^{2}$, Miloš Milovančević ${ }^{1}$ \\ ${ }^{1}$ Faculty of Mechanical Engineering, University of Niš, Serbia \\ ${ }^{2}$ Faculty of Engineering, University of Rijeka, Croatia
}

\begin{abstract}
The objective of this study is the application of multi-criteria optimization to the two-carrier two-speed planetary gear trains. In order to determine mathematical model of multi-criteria optimization, variables, objective functions and conditions should be determined. The subject of the paper is two-carrier two-speed planetary gears with brakes on single shafts. Apart from the determination of the set of the Pareto optimal solutions, the weighted coefficient method for choosing an optimal solution from this set is also included in the mathematical model.
\end{abstract}

Key Words: Multi-criteria Optimization, Planetary Gear Train, Two-Speed Planetary Gear Trains, Pareto optimal solutions

\section{INTRODUCTION}

Multi-criteria optimization problems are very common in many scientific and technical solutions. The optimization of gear trains as concrete technical systems implies complex mathematical model that has to describe real system operation in real circumstances.

Planetary gear trains (PGT) are a type of gear trains with many advantages. Since that their application is increasing in mechanical engineering and conveyor systems as single stage and multi-stage. Multi-stage planetary gear trains are obtained from single stage gear trains by linking one or two planetary units shafts.

Adequate design of PGT could be used as (multiple speed) gearboxes. Significant application as gearboxes has two-carrier planetary gear trains which consist of two coupled and four external shafts and enable two-speed transmissions [1]. There is a significant number of possible schemes of these transmissions [1-6]. Some transmissions structures could be used as two-speed transmissions by applying convenient brakes layout [1].

Received March 07, 2016 / Accepted September 20, 2016

Corresponding author: Jelena Stefanović-Marinović

Faculty of Mechanical Engineering, University of Niš, Aleksandra Medvedeva 14, 18000 Niš, Serbia

E-mail: jelenas@masfak.ni.ac.rs 
There are not many papers focus on the application of multi-criteria optimization to gear transmissions, especially planetary gear transmissions. Some papers are specified below.

The multi-objective optimization approach, based on the concept of Pareto optimality, is used in order to design helical gears [7]. The choice of the best parameters optimization is the important step in the process of obtaining the required gear quality and the optimization of the designing process itself [8].

Planetary gear transmissions are the subject of some papers in which the research relevant to the optimizations given. Arnaudow and Karaivanov [3] present a simple, descriptive and easy-to-handle method for investigating the transmission ratio, the internal power flows and the efficiency of complex multi-planetary gearings.

This paper provides an optimization of the two-carrier two-speed planetary gears with brakes on single shafts. For the numerical example of multi-criteria optimization application, the input data suitable for the usage at the fishing boat gearbox is chosen. Apart from the determination of the set of the Pareto optimal solutions, the weighted coefficient method is applied for choosing the optimal solution.

\section{MATHEMATICAL MODEL FOR PLANETARY GEAR TRAIN OPTIMIZATION}

In this paper multi-criteria optimization is applied to the two-carrier two-speed planetary gear trains with brakes on single shafts. These compound planetary gear trains consist of the basic type of planetary gear train.

The basic type of a planetary gear train (PGT) is a design which has a central sun gear (external gearing - 1), central ring gear (internal gearing - 3), planets (satellites - 2) and carrier (h), shown in Fig.1. Planets are in simultaneous contact with the sun gear and the ring gear. The multi-criteria optimization is limited to geared pairs.

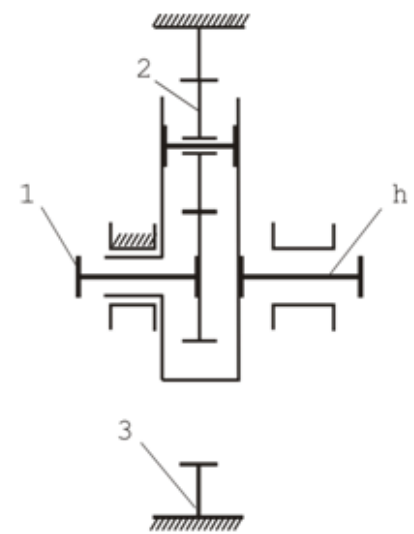

Fig. 1 Basic type of planetary gear train (1- sun gear; 2 - planet; 3 - ring gear; $\mathrm{h}$ - carrier)

The process of finding the optimal solution starts by defining a mathematical model [9]. The complete mathematical model of the basic type of a PGT is described in the aforementioned paper and will also be briefly presented in this section.

The mathematical model is defined by the variables, objective functions and conditions required for the proper functioning of a system expressed by the functional constraints. 


\subsection{Variables}

Under the mathematical model definition, it is necessary to determine the variables since each objective function is the function of several parameters.

In this paper, the following variables are considered: the number of teeth of the central sun gear $z_{1}$, the number of teeth of planets $z_{2}$, the number of teeth of the ring gear $z_{3}$, the number of planets $n_{w}$, the gear module $m_{n}$ and the face width $b$.

The optimization variables are of the mixed type: the numbers of gear teeth $\left(z_{1}, z_{2}, z_{3}\right)$ are integers, positive and negative, the number of planets $\left(n_{w}\right)$ is a discrete value, the module $\left(m_{n}\right)$ is a discrete standard value (acc. to ISO 54 (DIN 780)), while the face width $(b)$ is a continual variable. The numbers of gear teeth and the number of planets are non-dimensional values, while the module and the facewidth are given in millimeters.

\subsection{Objective functions}

In this model, the following characteristics are chosen for objective functions of a planetary gear train: volume, mass, efficiency and production cost of gear pairs.

The volume of gear pairs is used as an overall dimension expression and the approximation of the gear by the cylinder volume with the diameter equal to the pitch diameter and the height equal to the face width. The fact that planets are inside the ring gear makes it possible for the gear volume to be expressed by:

$$
V=\frac{\pi}{4} \cdot b \cdot\left(\frac{m_{n} \cdot z_{3}}{\cos \boldsymbol{\beta}} \cdot \frac{\cos \boldsymbol{\alpha}_{t}}{\cos \boldsymbol{\alpha}_{w t 23}}\right)^{2}
$$

where $\boldsymbol{\alpha}_{t}$ is the transverse pressure angle, $\boldsymbol{\alpha}_{w t 23}$ is the working transverse pressure angle for the pair 2-3 and $\boldsymbol{\beta}$ is the helix angle at the pitch diameter.

Mass is determined as the sum of all gear masses in a gear train. Since the mass of a particular gear is determined as gear volume multiplied by the density of gear material, this criterion receives the final expression, given by Eq. (2):

$m=0.25 \cdot \boldsymbol{\pi} \cdot b \cdot \boldsymbol{\rho} \cdot \frac{m_{n}^{2}}{\cos ^{2} \boldsymbol{\beta}}\left(k_{1} \cdot z_{1}^{2} \frac{\cos ^{2} \boldsymbol{\alpha}_{t}}{\cos ^{2} \boldsymbol{\alpha}_{w t 12}}+n_{w} \cdot k_{2} \cdot z_{2}^{2} \frac{\cos ^{2} \boldsymbol{\alpha}_{t}}{\cos ^{2} \boldsymbol{\alpha}_{w t 12}}+k_{3} \cdot z_{3}^{2} \frac{\cos ^{2} \boldsymbol{\alpha}_{t}}{\cos ^{2} \boldsymbol{\alpha}_{w t 23}}\right)$

Efficiency is one of the most important criteria for the design and evaluation of the construction quality. Power losses in planetary transmissions consist of losses in the gear contact, losses in bearings and losses due to oil viscosity. The calculation of the gear transmission efficiency is generally confined to losses depending on friction on tooth sides, i.e. on the calculation of contact power losses [9-11]. Then, the following expression for efficiency is considered:

$$
\eta=\frac{1-i_{0} \cdot \eta_{0}}{1-\eta_{0}}
$$

where $\boldsymbol{\eta}_{0}$ is the efficiency when the carrier is immovable

$$
\eta_{0}=1-\frac{z_{3}}{z_{3}-z_{1}}\left(\frac{0.15}{z_{1}}+\frac{0.35}{z_{2}}+\frac{0.20}{z_{3}}\right)
$$

and $i_{0}$ is basic transmission ratio $i_{0}=z_{3} / z_{1}$. 
Economic demands must also be taken into consideration in the techno-economical optimization. First, these demands are related to production costs. These costs consist of the production material and the production process costs. The time needed for the production of gears is taken as a measure of the production costs and as an economic factor. This function is then determined as a sum of time periods needed for the production of the central sun gear $\left(T_{P 1}\right)$, the planets $\left(T_{P 2}\right)$ and the ring gear $\left(T_{P 3}\right)$, i.e.

$$
F_{T}=T_{P 1}+n_{w} \cdot T_{P 2}+T_{P 3}
$$

Production times are determined according to the technologies of Fette, Lorenc and Höfler [12].

\subsection{Functional constraints}

Planetary gear trains represent a specific group of gear trains. Therefore, there are numerous exceptions that need to be taken into account for these transmissions to function correctly compared with classical gear transmissions. The exceptions presented in this article are related to mounting conditions, geometrical conditions and strength conditions.

The mounting conditions comprise the condition of coaxiality, the condition of adjacency and the condition of conjunction [13].

Geometrical conditions relate to the undercutting and profile interference, the ratio of the pressure angle to the working transverse pressure angle, the tooth thickness and the space width, the transverse contact ratio value, the sliding speeds, the ratio of the pinion face width to the pinion reference diameter, etc. These conditions are ensured in accordance with the actual standards (ISO TC 60 list of standards 090915).

The strength conditions, safety factors for bending strength and surface durability of each gear, are provided according to ISO 6336-1 to ISO 6336-3 [14].

\subsection{Optimization procedure}

The base of the optimization process of PGT presented in this paper is the comparison of solutions with different parameters in the same conditions and the selection of the best variant. The optimization process begins with generating all solutions for the assigned input data. For the given input data (transmission ratio, input number of revolution, input torque, service life in hours, application factor, accuracy grade (Q-DIN3961)), all 6-tuples of design parameters $\left(z_{1}, z_{2}, z_{3}, n_{w}, m_{n}, b\right)$ satisfying the functional constraints are generated and the values of the objective functions for every 6-tuple are computed. These 6-tuples form a set of feasible solutions. Based on the established objective functions and constraints, an optimal solution is selected, determined by variables.

The mathematical model of nonlinear multicriteria problem can be formulated as follows:

$$
\begin{aligned}
& \max \left\{f_{1}(x), f_{2}(x), \ldots \ldots, f_{k}(x)\right\} \\
& \text { subject to } x \in S
\end{aligned}
$$

Here, $f_{1}(x), \ldots ., f_{k}(x)$ are objective functions and $x=\left(x_{1}, \ldots \ldots, x_{n}\right)$ is the vector of decision variables and $S$ is the set of feasible solutions. Every point $x \in S$ is mapped to the point $\left(f_{1}(x)\right.$, $\left.f_{2}(x), \ldots f_{k}(\mathrm{x})\right)$ in $k$-dimensional objective space. Therefore, one can observe the objective set:

$$
F=\left\{\left(\left(f_{1}(x), f_{2}(x), \ldots \ldots, f_{k}(x)\right) \mid x \in S\right\}\right.
$$


The notation "max" determines simultaneous maximization of all the objective functions. If any objective function is to be minimized, minimization of the function $f_{i}(x)$ is equivalent to the maximization of the function $-f_{i}(x)$. According to the structure of the feasible set $S$, there exist discrete multi-criteria optimization problems. In our planetary gears problem, six decision variables exist, corresponding to the basic design parameters: $x=\left(x_{1}, x_{2}, x_{3}, x_{4}, x_{5}, x_{6}\right)$ $=\left(z_{1}, z_{2}, z_{3}, n_{w}, m_{n}, b\right)$. Furthermore, there are four objective functions: volume $V(x)$, mass $m(x)$, efficiency $\boldsymbol{\eta}(x)$ and production costs $T(x)$ :

$$
\begin{gathered}
f_{1}(x)=-V(x), f_{2}(x)=-m(x), \\
f_{3}(x)=\eta(x), f_{4}(x)=-T(x)
\end{gathered}
$$

Then, mathematical model of nonlinear multi-criteria problem in concrete task, can be formulated as follows:

$$
\begin{aligned}
& \max \left\{f_{1}(x), f_{2}(x), f_{3}(x), f_{4}(x)\right\} \\
& \text { subject to } x \in S
\end{aligned}
$$

It is often useful to know the best possible values for each objective function. These values form a so-called ideal point $f^{*}=\left(f_{1}^{*}, \ldots ., f_{k}^{*}\right)$ in the objective space. Its components are computed as:

$$
\begin{aligned}
& f_{i}^{*}=\max f_{i}(x), i=1, \ldots . k \\
& x \in S
\end{aligned}
$$

As it can be seen from the definition, multi-criteria optimization problems are mathematically ill-defined. The most important criterion for selecting these "equally good" solutions is the Pareto optimality concept: Solution $x \in S$ is Pareto optimal if there is no solution $y \in S$ such that holds $f_{i}(x) \leq f_{i}(y)$ for all $i=1, \ldots \ldots, n$ and for at least one index $i$ holds strict inequality, i.e. $f_{i}(x)<f_{i}(y)$. Determination of the Pareto optimal solutions set is the first step in optimal solution finding. Next step is optimal solution choice from Pareto solutions set. In this model weighted coefficients method is applied for choosing optimal solution from Pareto solutions.

\subsection{Weighted coefficients method}

In this method the following scalarized problem is constructed:

$$
\begin{aligned}
& \max f^{M}(x)=w_{1} \cdot f_{1}^{0}(x)+\ldots \ldots+w_{m} \cdot f_{m}^{0}(x) \\
& \text { s.t. } x \in S
\end{aligned}
$$

Here, the weighted coefficients (or weights) $w_{i}$ are positive real numbers and $f_{i}^{0}(x)=$ $\left(f_{i}^{0}\right)^{-1} f_{i}(x)$ are normalized objective functions where $f_{i}^{0}$ are normalizing coefficients. In this approach, the components of the ideal point $f^{*}=\left(f_{1}{ }^{*}, f_{2}{ }^{*}, f_{3}{ }^{*}, f_{4}{ }^{*}\right)$ are used as normalizing coefficients, i.e. $f_{i}^{0}=f_{i}^{*}$ for $i=1,2,3,4$. Therefore, absolute values of all objective functions are between 0 and 1 , which simplifies the choice of the weighted coefficients. All solutions obtained by this method are Pareto optimal [9]. The weighted coefficients method has very clear physical meaning and experience in application on technical systems optimization. 
This model is suitable in the case of priority functions existence, also in the case of equal priority functions [12].

A shortened algorithm for the complete optimization procedure is shown in Fig. 2. The complete optimization procedure is implemented in the PlanGears software.
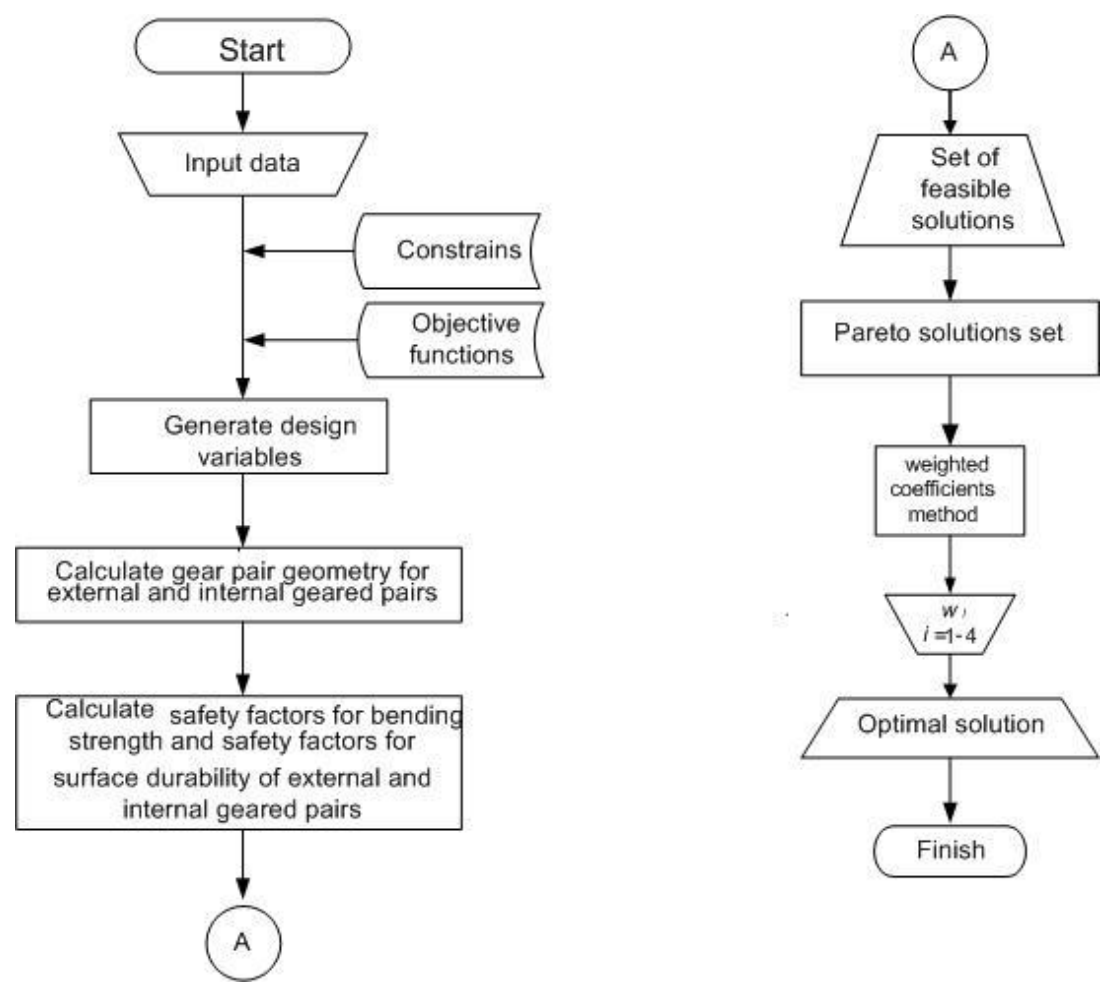

Fig. 2 Shortened algorithm for the optimization procedure

\section{TwO-SPEED Planetary Gear TRains}

Speed change under load is advantage, and in some occasions request of mechanical system (e.g. machine tools, cranes etc.). Two-speed two-carrier planetary gears which consist of two coupled and four external shafts and have brakes on single shafts could be use in these systems. A special type of mechanism is obtained by settings the brakes on two external shafts that allow energy flow managing throw the transmission and transmission ratio changing. The layout of brakes at these compound gear transmissions has many possibilities $[15,16]$.

In Fig. 3 ideal torque ratios and torque ratios, as well as Wolf-Arnaudov's symbol, of the basic type of PGT are pointed. The carrier shaft is summary element, since by carrier stopping negative transmission ratio is obtained. 
Prerequisite:

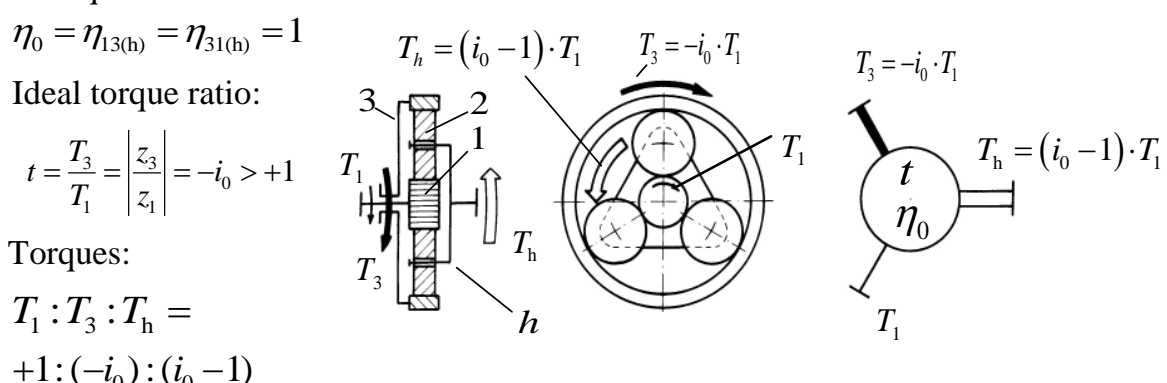

$+1:\left(-i_{0}\right):\left(i_{0}-1\right)$

Fig. 3 Torque ratios and Wolf-Arnandov's symbol of the basic type of PGT

\subsection{Structures of compound two-carrier PGT and their labeling}

A Planetary gear train with four external shafts is shown in Fig. 4.

Two component trains can be joined in a whole in 12 different ways, called the planetary gear train with four external shafts [16]. To each of 12 structural schemes an alphanumerical label (S11...S56) is attached, which indicates the ways of connecting the shafts of the main elements of both component trains (Fig. 5). In every presented scheme it is possible to put brakes as well as the driving and the operating machine on external shafts in 12

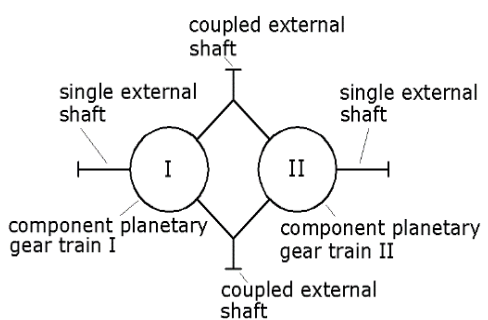

Fig. 4 Planetary gear train with four external shafts (compound train) different ways (V1...V12), which will be here called layout variants (Fig. 6).

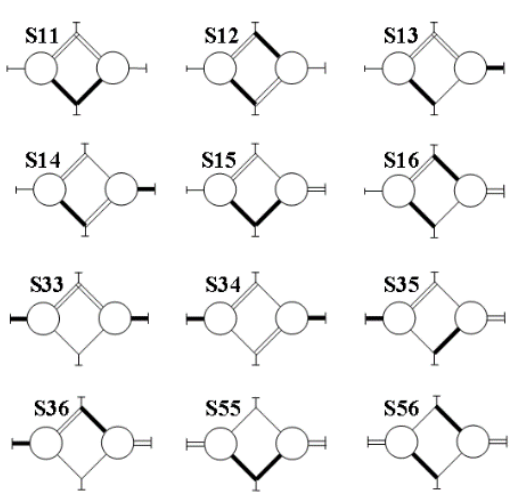

Fig. 5 Systematization of all schemes of two-carrier planetary gear train with four external shafts

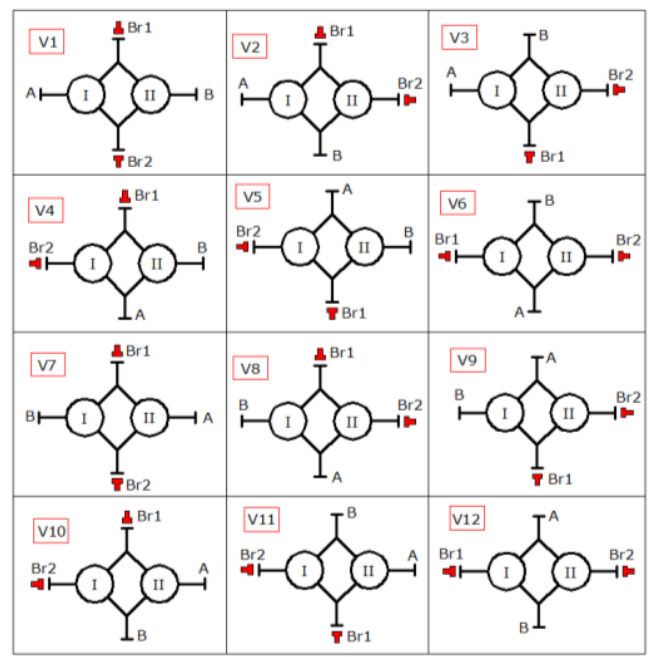

Fig. 6 Systematization of all layout variants 


\subsection{Analysis of the operations of compound trains with different layout variants}

By situating the brakes on two shafts a braking system is obtained in which the alternating activation of the brakes shifts the power flow through the planetary gear train, which causes a change in the transmission ratio [1, 3, 6, 15, 16, 17]. Kudrjavtsev and Kirduashev [2] present 15 kinematic schemes of the considered type and achievable values of transmission ratios and efficiencies of both gears. Troha et al. [1, 15, 17] present the computer program for the selection of an optimal variant of similar multi-speed planetary gear trains including shifting capabilities charts for all possible two-speed planetary gear trains.

The considered compound trains can be divided into three different groups depending on the layout of brakes on the shafts. The first group consists of the compound trains with brakes on the coupled shafts. The second group consists of the compound trains with brakes on the single shafts. The third group consists of the compound trains with brakes on the coupled and the single shaft. All compound trains within separate groups have some specific common characteristics [16].

The compound train with brakes on the single shafts (layout variants V6 and V12) is symbolically shown in Fig. 7. When the left brake is turned on, power is transmitted through the left component train (component train I), and when the right brake is turned on, power is transmitted through the right component train (component train II). Input and output of power are on the coupled shafts. In this case, whatever the brake is turned on, only one component train operates actively while the other operates idly. Therefore, the transmission ratios of the compound train are equal to the transmission ratios that the component trains accomplish.

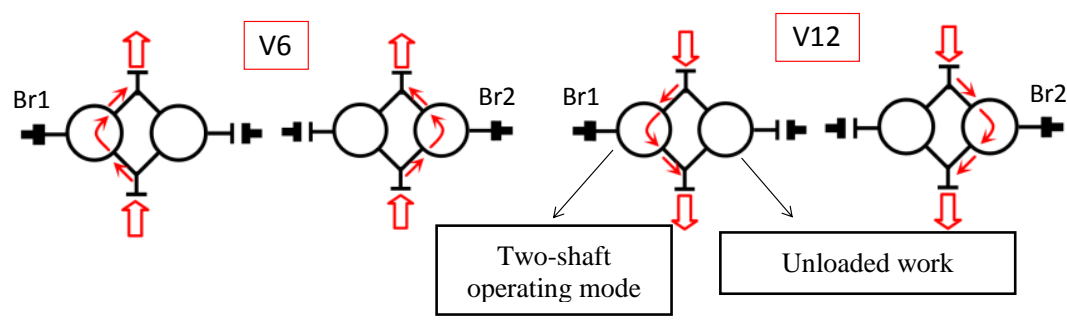

Fig. 7 Possible power flows through the train with brakes on the single shafts

Each variant has its own characteristics that determine the possibilities of converting. It could be presumed that some variants work in both speeds lake reducers and multipliers, while some other variants work like reducers with one speed and like multiplier with other speed. Also, some variants change direction of rotation when change speed, while some other variants keep the direction of rotation during speed changing. Transmission ratio of each planetary gear train unit depends only on basic transmission ratio (ideal torque ratio).

Some of these variants have very interesting kinematic characteristics from the point of the practice. For example, transmission variant S36V6 which changes the direction of rotation during speed changing is suitable for application in machine tools where exist one working motion with considerable resistances and low speed and other working motion during returning to the starting position with great speed. Transmissions with brakes on single shafts have their own constraints. Considerable constraint is transmission ratio range: between 0.0769 and 13. If transmission ratio besides this range is need, usage of transmissions with brakes on coupled shafts or on coupled and single shaft is recommendation. 


\section{RESULTS AND DISCUSSION}

For the example of multi-criteria optimization application variant S36V6 is chosen (Figs. 8 and 9). This type of transmission has considerable application at the systems which need transmission ratio changes under load. For instance, it is applicable as a drive of fishing boats propellers. In that case the transmission works with transmission ratio $i=4$ in one way and with transmission ratio $i=-4$ in the other way. The transmission is situated on the propeller shaft between engine and propeller. These data is adopted for the input data for optimal solution choice.

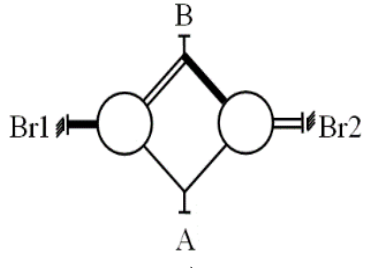

a)

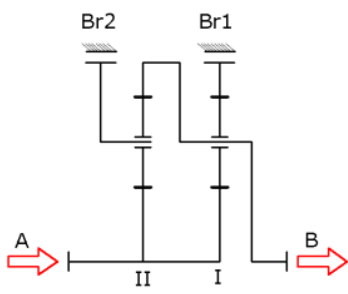

b)

Fig. 8 Symbolic review of transmission composition (a), kinematic scheme (b)

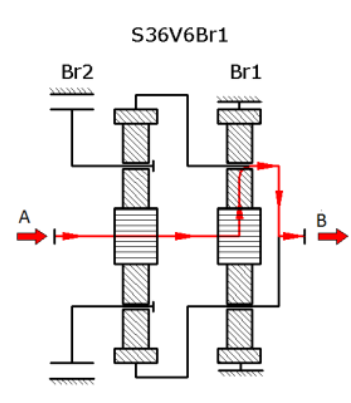

a)

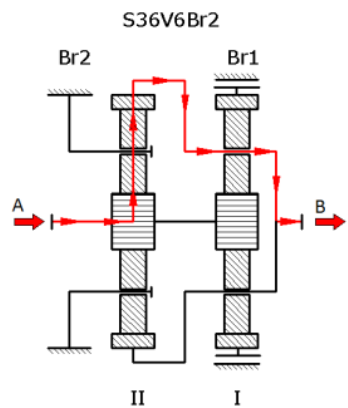

b)

Fig. 9 Power flow throws the transmission when the brake $\mathrm{Br} 1$ is activated (a) and when the brake $\mathrm{Br} 2$ is activated (b)

\subsection{The first stage of compound gear train (I)}

When the brake $\mathrm{Br}$ is activated and the brake $\mathrm{Br} 2$ inactivated the ring gear in first stage is immovable. The input of the system is A, and sun gear of the first stage and sun gear of the second stage have the same number of revolution. Since the ring gear in the first stage (3I) is reactive, the power is transmitted to the carrier of the first stage (HI) and than to the output B. The second stage works at the three-shaft mode, but no resistance, i.e. at idle. The first stage is determined in this mode.

The next input data is chosen for the multi-criteria optimization application: $i_{0}=-3.0$, $n_{i n}=1800 \mathrm{~min}^{-1}, T_{i n}=3119.34 \mathrm{Nm}(P=588 \mathrm{~kW}), L=8000 \mathrm{~h}, K_{A}=1.25$, IT7, for all gears, material $z_{1} /$ material $z_{2}$ /material $z_{3}=20 \mathrm{MoCr} 4 / 20 \mathrm{MoCr} 4 / 34 \mathrm{CrNiMo6}, S_{\text {Hmin }}=1.1, S_{F \min }=1.2$, $\Delta i=3 \%, z_{1}=15 \div 30$. 
The feasible set consists of 7713 solutions. The number of Pareto solutions is 20. By application weighted coefficient method with weighted coefficient: $w_{1}=0.5, w_{2}=0.0, w_{3}=0.0$, $w_{4}=0.5$ the solution shown in Table 1 is obtained, with a set of objective functions values shown in Table 2.

Table 1 Optimal solution obtained by weighted coefficient method

\begin{tabular}{cccccc}
\hline \multicolumn{7}{c}{ Variable values } \\
\hline$x_{1}=z_{1}$ & $x_{2}=z_{2}$ & $x_{3}=z_{3}$ & $x_{4}=n_{w}$ & $x_{5}=m_{n}$ & $x_{6}=b$ \\
23 & 23 & -67 & 5 & 5 & 34 \\
\hline
\end{tabular}

Table 2 Objective function for solution shown in table 2

\begin{tabular}{cccc}
\hline$f_{1}\left[\mathrm{~mm}^{3}\right]$ & $f_{2}[\mathrm{~kg}]$ & $f_{3}$ & $f_{4}[\mathrm{~min}]$ \\
\hline 3218716.637 & 18.476 & 0.986 & 257.25 \\
\hline
\end{tabular}

\subsection{The second stage of compound gear train (II)}

When the brake $\mathrm{Br} 2$ is activated and the brake $\mathrm{Br} 1$ inactivated, sun gear of the first stage and sun gear of the second stage rotate, but the carrier of the second stage is reactive and power transmitted through the sun gear of second stage and ring gear of second stage to the output $\mathrm{B}$. The second stage is determined in this mode.

If the carrier is immovable, the required transmission ratio is equal to the basic transmission ratio (ideal torque ratio). Taking into account the dependence between the basic transmission ratio and the transmission ratio with the immovable transmission ratio, the next input data is chosen for the multi-criteria optimization application in this stage: $i_{0}=-4.0$, $n_{i n}=1800 \mathrm{~min}^{-1}, T_{i n}=3119.34 \mathrm{Nm}, L=8000 \mathrm{~h}, K_{A}=1.25$, IT7 for all gears, material $z_{1} /$ material $z_{2} /$ material $z_{3}=20 \mathrm{MoCr} 4 / 20 \mathrm{MoCr} 4 / 34 \mathrm{CrNiMo6}, S_{\text {Hmin }}=1.1, S_{\text {Fmin }}=1.2, \Delta i=3 \%, z_{1}=15 \div 30$.

The feasible set consists of 10466 solutions. The number of Pareto solutions is 73 . By application weighted coefficient method with weighted coefficient: $w_{1}=0.5, w_{2}=0.0, w_{3}=0.0$, $w_{4}=0.5$ the solution shown in Table 3 is obtained, with a set of objective functions values shown in Table 4.

Table 3 Optimal solution obtained by weighted coefficient method

\begin{tabular}{cccccc}
\hline \multicolumn{7}{c}{ Variable values } \\
\hline$x_{1}=z_{1}$ & $x_{2}=z_{2}$ & $x_{3}=z_{3}$ & $x_{4}=n_{w}$ & $x_{5}=m_{n}$ & $x_{6}=b$ \\
20 & 29 & -79 & 3 & 5.5 & 44 \\
\hline
\end{tabular}

Table 4 Objective function for solution shown in table 4

\begin{tabular}{cccc}
\hline$f_{1}\left[\mathrm{~mm}^{3}\right]$ & $f_{2}[\mathrm{~kg}]$ & $f_{3}$ & $f_{4}[\mathrm{~min}]$ \\
\hline 6476760.79 & 31.04 & 0.986 & 274.597 \\
\hline
\end{tabular}

It can be concluded that the Pareto optimality concept as the criterion for selecting equally good solution makes sense to apply to compound PGT according to these criteria. 


\section{CONCLUSIONS}

In this paper, an original model for multi-criteria optimization of two-carrier two-speed planetary gear trains with brakes on single shafts has been presented. These compound gear trains consist of two basic type of planetary gear trains and have considerable application at the systems which need transmission ratio changes under load. The optimal solutions of chosen variant of these gear trains are obtained for the both transmission ratios. The weight coefficient method is used for choosing the optimal solution from the Pareto optimal set.

This approach is original in the planetary gear train optimization and can be successfully used for the basic planetary gear train type and compound gear trains consist of basic type, as is shown in this application. The results obtained in this way are in accordance with the literature on technical system optimization and indicate a good choice of the applied methods. Furthermore, this approach indicates a possibility for application to other planetary gear train types.

\section{REFERENCES}

1. Troha, S., 2011, Analysis of a planetary change gear train's variants, (in Croatian), PhD Thesis, University of Rijeka, Engineering Faculty, Rijeka, Croatia., 395 p.

2. Kudrjavtsev, V. N., Kirdyiashev, I. N., 1977, Planetary gears, (in Russian), Handbook, Mashinostroenie, Leningrad., $535 \mathrm{p}$.

3. Arnaudow, K., Karaivanov, D., 2005, Systematik, Eigenschaften und Möglichkeiten von zusammengesetzten Mehrsteg-Planetengetrieben, Antriebstechnik, 5, pp. 58-65.

4. Ivanov, A., N., 1990, Evaluation of diametric dimensions of planetary gearboxes in the design phase, (in Russian),Vestnik mashinostroenie, 7, pp. 16-19.

5. Lechner, G., Naunheimer, H.,1999, Automotive transmissions, Springer-Verlag, Heidelberg, 438 p.

6. Jelaska, D., 2012, Gears and Gear Drives, University of Split, Croatia, 444 p.

7. Tudose, L., Buiga, O., Jucan, D., Stefanache, C., 2008, Multi-objective optimization in helical gears design, Proc. The Fifth International Symposium about Design in Mechanical Engineering KOD 2008, pp. 77-84, Novi Sad, Serbia.

8. Tkachev, A., Goldfarb, V., 2009, The concept of optimal design for spur and helical gears, Proc. The 3rd International Conference Power Transmissions 2009, pp.59-62, Kallithea, Greece.

9. Stefanović-Marinović, J., Petković, M., Stanimirović I., Milovančević, M., 2011, A model of planetary gear multicriteria optimization, Transactions of FAMENA, 35(4), pp. 21-34

10. Sriatih, A., Yedukondalu G., Jagadeesh, A., 2011, Mechanical efficiency of planetary gear trains: An Estimate, Mechanical Engineering Research, 1(1), pp. 97-102.

11. Del Castillo, J.M., 2002, The analytical expression of the efficiency of planetary gear trains, Mechanism and Machine Theory, 37, pp. 197-214.

12. Stefanović-Marinović, J., 2008, Multicriterion optimization of planetary power transmission gear pairs, (in Serbian), PhD Thesis, University of Niš, Faculty of Mechanical Engineering, Niš, Serbia, 291 p.

13. Niemann G., Winter H., 1989, Maschinenelemente, Band II, Springer-Verlag Berlin, 376 p.

14. International Organization for Standardization, 2006, International standard ISO 6336-2, Calculation of load capacity of spur and helical gears.

15. Troha, S., Lovrin , N., Milovančević, M., 2012, Selection of the two-carrier shifting planetary gear train controlled by clutches and brakes, Transactions of FAMENA, 36(3), pp. 1-12.

16. Troha, S., Žigulić, R., Karaivanov, D., 2014, Kinematic operating modes of two-speed two-carrier planetary gear trains with four external shafts, Transactions of FAMENA, 38(1), pp. 63-76.

17. Troha S., Petrov P., Karaivanov, D., 2009, Regarding the optimization of coupled two carrier planetary gears with two coupled and four external shafts, Machinebuilding and electrical engineering, 1, pp. 49-56. 